\title{
Porous Poly(Acrylic Acid) from High Internal Phase Emulsion: Effects of Emulsification Parameters on Porous Structure
}

\author{
Ranran $\mathrm{ZHANG}^{1, \mathrm{a}}$, Yun $\mathrm{ZHU}^{2, \mathrm{~b}}$, Shengmiao $\mathrm{ZHANG}^{3,{ }^{*} \mathrm{c}}$ and Jianding $\mathrm{CHEN}^{4, \mathrm{~d}}$ \\ 1234 School of Materials Science and Engineering, East China University of Science and Technology, \\ Shanghai 200237, China; \\ a 845208110@qq.com ${ }^{1},{ }^{b}$ zhuyun@ecust.edu.cn, ${ }^{\mathrm{c}}$ shmzhang@ecust.edu.cn, ${ }^{\mathrm{d}}$ \\ jiandingchen@ecust.edu.cn
}

\begin{abstract}
Highly porous poly (acrylic acid) (P(AA)) with tunable pore structure was prepared through polymerizing of acrylic acid in the aqueous phase of an oil-in-water (O/W) high internal phase emulsion (HIPE). Compared with the conventional O/W HIPE normally using toluene or hexane as internal phase, the introducing paraffin, which have a much higher viscosity than toluene or hexane, caused the HIPEs herein could be stabilized by Tween 60 of low to $0.5 \mathrm{wt} \%$. It was found the amount of liquid paraffin played a key role for the stability of the emulsion and the morphology of the resulting porous materials. Moreover, the average void size and the interconnectivity degree of the porous materials could also be tailored by altering the acrylic acid and surfactant concentration in aqueous phase.
\end{abstract}

\section{Introduction}

High internal phase emulsion (HIPE) is defined as the droplets (internal) phase in the emulsion volume fraction is higher than $74.05 \%$. HIPE has been known earlier and has many applications in the fields of cosmetics and food industry[1]. Polymerized high internal phase emulsion, or PolyHIPEs, is the material resulting from the polymerization of HIPE continuous phase. In traditional HIPE, the concentration of surfactant is always very high, normally $5-50 \%$ relative to the continuous phase, which is not environmental friendly and is the most cost factor. For example, porous poly (acrylic acid) (PAA) from oil-in-water $(\mathrm{O} / \mathrm{W}) \mathrm{HIPE}$ with the concentration of surfactant up to $21 \mathrm{wt} \%$ was prepared[2]. Although great efforts have been done to eliminate or reducing the amount of the surfactant in the preparation of HIPEs, such as particle-stabilized[3, 4] and CTAB-stabilized HIPEs[5], HIPE stabilized by non-ionic surfactant of low concentration is still highly desired, due to the easy removal of the residue surfactant from the resulting porous materials.

In this study, by introducing paraffin as internal phase, which have a much higher viscosity than toluene or hexane that is normally used as the internal phase of O/W HIPE, caused the HIPEs herein could be stabilized by Tween 60 of low to $0.5 \mathrm{wt} \%$. Polymerizing acrylic acid in these HIPE, a series of PAA were obtained. These porous PAA had a

\footnotetext{
*Corresponding author:845208110@qq.com
} 
controlled and well-defined open porous structure and were $\mathrm{pH}$-sensitive.

\section{Experiment Section}

\subsection{Materials}

Acrylic acid (AA, Shanghai Lingfeng Chemical Reagent Ltd.Co.) was purified by vacuum distillation.N,N-methylenebis acrylamide ( MBAM, 98\%, Aldrich), Tween60 (Sinopharm Chemical Reagent Co., Ltd), Tetramethylethylenediamine (TEMED, 99\%, Aladdin) and ammonium persulphate (APS, 98\%, Shanghai Lingfeng Chemical Reagent Ltd.Co.) are purified via recrystallization twice. Other materials were standard laboratory reagents and were used as received.Water was fresh deionized.

\subsection{Preparation and Characterization of HIPE and PolyHIPEs}

Acrylic acid and the cross-linker (MBAM) $(20: 1, \mathrm{~mol} / \mathrm{mol})$ was added to an aqueous solution of Tween 60. After the monomers were dissolved, the initiator APS was added to the acrylic acid solution.Then liquid paraffin was added dropwise to the monomer solution under constant stirring with a magnetic stirrer at $600 \mathrm{rpm}$. The stirring was continued for 5 min after the addition of liquid paraffin was completed. A reducing agent TEMED was then added to the mixture, and after 3 min of additional stirring, the emulsion was transferred to the mold and cured at $50{ }^{\circ} \mathrm{C}$ for $24 \mathrm{~h}$. The resulting monolith was purified via a Soxhlet extraction with cycloheaxane for $24 \mathrm{~h}$. The polyHIPEs were dried in a freezing dryer, then white materials were obtained.

TABLE 1 COMPOSITION OF PAA HIPES AND THE RESULTING POROUS MATERIALS ${ }^{A}$.

${ }^{a}$ The concentration of MBAM relative to the aqueous phase was $2 \mathrm{wt} \% .{ }^{b}$ AA concentration in the aqueous phase. ${ }^{c}$ Tween 60 concentration in the aqueous phase. ${ }^{d}$ The internal phase volume fraction of HIPEs. ${ }^{e}$ The average void diameter of porous PAA.

\begin{tabular}{ccccc}
\hline $\begin{array}{c}\text { Sample } \\
\text { ID }\end{array}$ & $\begin{array}{c}\mathrm{AA}^{b} \\
{[\mathrm{wt} \%]}\end{array}$ & $\begin{array}{c}\text { Tween60 }^{c} \\
{[\mathrm{wt} \%]}\end{array}$ & $\begin{array}{c}\text { Internal phase volume } \\
\text { fraction }\end{array}$ & $\begin{array}{c}\mathrm{D}^{e} \\
{[\%]}\end{array}$ \\
\hline 1 & 20 & 1 & 80 & $4 \mu \mathrm{m}]$ \\
2 & 30 & 1 & 80 & 20.9 \\
3 & 40 & 1 & 80 & 14.0 \\
4 & 30 & 0.5 & 80 & 61.9 \\
5 & 30 & 2 & 80 & 15.7 \\
6 & 30 & 1 & 75 & 43.6 \\
7 & 30 & 1 & 90 & 19.5 \\
\hline
\end{tabular}

For SEM analysis, the samples were cut with surgical blade and coated with gold in vacuum before observation. The average void diameters of the polyHIPEs were measured using an image analysis software Image J (NIH image). Average diameters measured in thisway are under estimating of the real values. Therefore it is necessary to introduce a statistical correction[6]. The average void diameter (D) of each polyHIPE material in this 
work was achieved as Eq. 1.

$$
D=\frac{2}{\sqrt{3}} \cdot d
$$

where $\mathrm{D}$ is the equatorial value of void diameter and dis the diameter value calculated from the SEM image. The interconnectivity degree I of the monolith was calculated by Eq. 2

$$
I=\frac{1}{4} \cdot N \cdot\left(\frac{\mathrm{r}}{D}\right)^{2}
$$

where $\mathrm{N}$ was the average number of interconnecting pores per void, $\mathrm{r}$ was the average interconnecting pore diameter. At least 50 voids were calculated for each sample.

Sample 3 was chosen to take the $\mathrm{pH}$-sensitive test for PAA porous material. Three monoliths of it were cut with basically identical size and quality and then were immersed into the water with different $\mathrm{pH}$ value, respectively. A digital camera was used to observe its size change. Specific procedures were described as following: weight of the dry monolith was called $\mathrm{M}_{0}$ and then it was immersed into the aqueous. The wet monolith was weighed in every ten minutes within $1 \mathrm{~h}$, named $\mathrm{M}_{1}$. Before weighing the wet monolith, the water on its surface was cleaned with filter paper. The cleaning and weighing must be finished quickly. And then got the water absorption multiple by Eq.3

$$
A=\frac{M_{1}-M_{0}}{M_{0}}
$$

Finally the curves of water absorption multiple vs time for the three monoliths were got.

\subsection{Results and Discussion}

As shown in Table 1, a series of HIPEs were prepared to investigate the effect of changing the AA and Tween 60 concentration in the aqueous phase as well as the liquid paraffin fraction on the average void size and the interconnectivity degree. 


\subsection{The Effect of the AA Concentration}
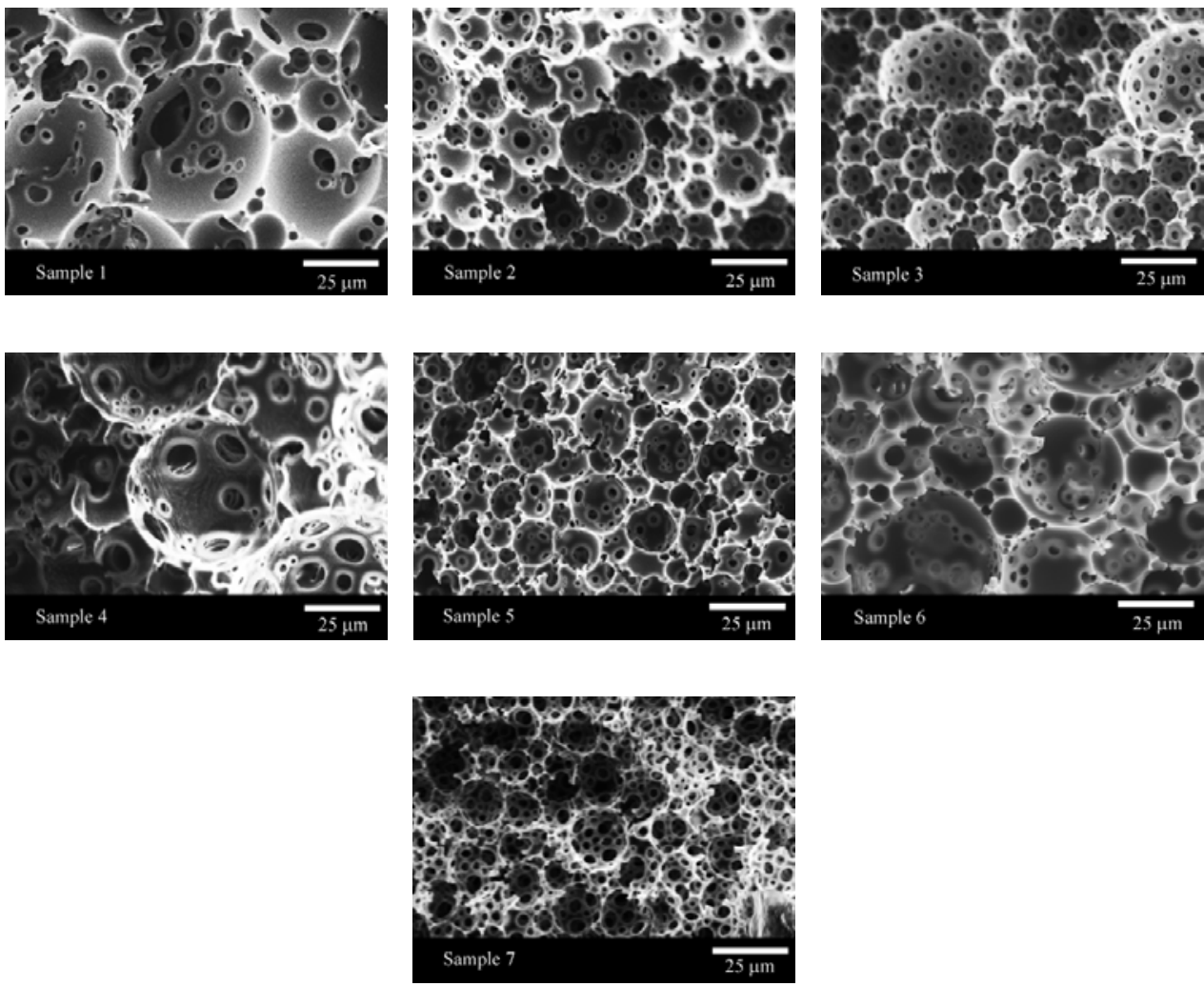

Fig. 1 SEM images of PAA porous materials from o/w HIPEs templates.

As shown in Table 1 and Fig. 1, when the monomer concentration was increased from 20 to $40 \mathrm{wt} \%$, the average void diameter of the PolyHIPEs decreased dramatically(Sample $1,2,3)$. Because when the AA concentration increased in the aqueous phase, the HIPE was easier to get a gel point during the polymerization of HIPE, which made the HIPEs more stable and then the emulsions trended to form smaller and more homogeneous drops, therefore caused a smaller void diameter since the void of the resulting porous materials was duplicated from the droplet[7]. Moreover, increasing the monomer concentration also decreased the interconnecting pore diameter and interconnectivity degree. This phenomenon resulted from the fact that the more stable emulsion, the smaller droplets and more droplets. So that the concentration of surfactant(Tween 60), considered as porogen for formation of interconnect pore[8], in each droplet decreased, which caused the decreasing of the average interconnecting pore diameter and the interconnectivity degree (Fig.2). 


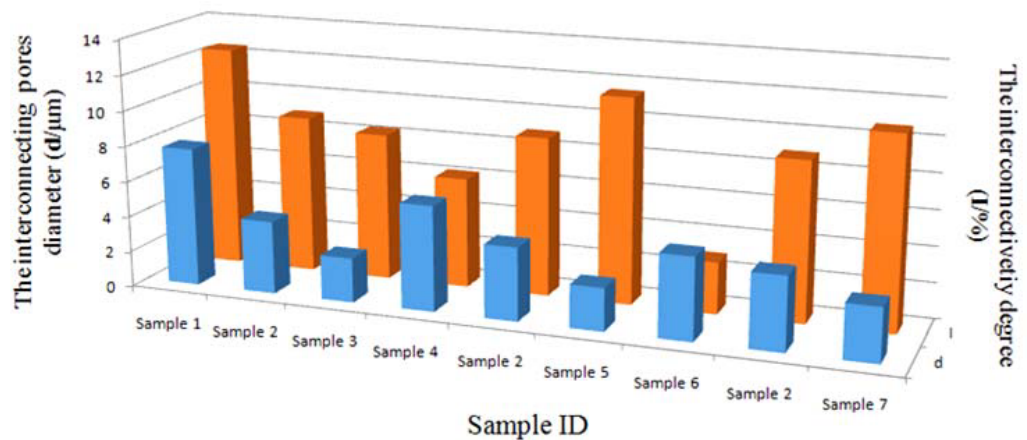

Fig.2 The interconnecting pores diameter and the interconnectivity degree of the Samples, the former was $\mathrm{d}$ and the back was I.

\subsection{The Effect of the Tween60 Concentration}

Fig. 1 (Sample 4, 2, 5) showed that when the concentration of Tween 60 in the aqueous phase increased, the void diameter and interconnecting pore diameter decreased, and the interconnectivity increased. This could be due to the fact that increasing Tween 60 concentration enhanced the HIPEs stability, which caused more and smaller droplets in the emulsion and, and consequently smaller void diameter of the resulting polyHIPE. While the void diameter decreased, the interconnecting pore diameter decreased as well, but the interconnectivity degree raised. This was because the surfactant involved not only to be stabilizer but also acted as the building block for the final polymeric materials wall. When its concentration increased, each emulsion drop carried more Tween 60 making more interconnecting pore in the resulting materials and raising the interconnectivity degree (Fig.2).

\subsection{The Effect of the Internal Fraction}

As shown Table 1 and Fig. 1, Sample 6, 2, 7, the void diameter decreased with an increase of internal phase fraction, which was different from the traditional polyHIPEs, in which the diameter increased with an increase of the internal phase fraction. In this study, the cause of this phenomenon was due to the usage of liquid paraffin as internal phase. Liquid paraffin has a higher viscosity compared with the AA aqueous solution that was used as continuous phase. When increased paraffin fraction of the HIPE, viscosity of mixture increase significantly. Then the emulsion viscosity increased, which enhanced its stability and decreased the emulsion droplet size. Thus the void diameter of final porous materials decreased. Moreover, increasing internal phase fraction also made the film between the neighboring droplets thinner, which was helpful to formation of the interconnected pore and finally resulted in higher interconnectivity degree (Fig.2).

\section{7pH-sensitive Property of Porous PAA}

In this study, the pH-sensitive property had been explored. As shown in Fig.2 a, the three monoliths were cut from the same sample(e. g. Sample 3 in Table 1 and Fig. 1), and it could be found that the shape and size of all three monoliths were nearly the same. As show in Fig. $1 \mathrm{~b}$ and c, it could be clearly observed that the porous PAA monoliths had a 
different swelling degree in the water with different $\mathrm{pH}$ value. As shown in Fig.4, at the beginning of the swelling process, the swelling rate was faster in a higher $\mathrm{pH}$ medium and thus the water absorption multiple was higher.The swelling equilibrium in both acid and alkali medium was got within $1 \mathrm{~h}$, however, the swelling equilibrium of the monolith immersed in the nearly neutral medium was got in $8 \mathrm{~h}$ and the final water absorption multiple of this sample was much higher than those of the other two samples.
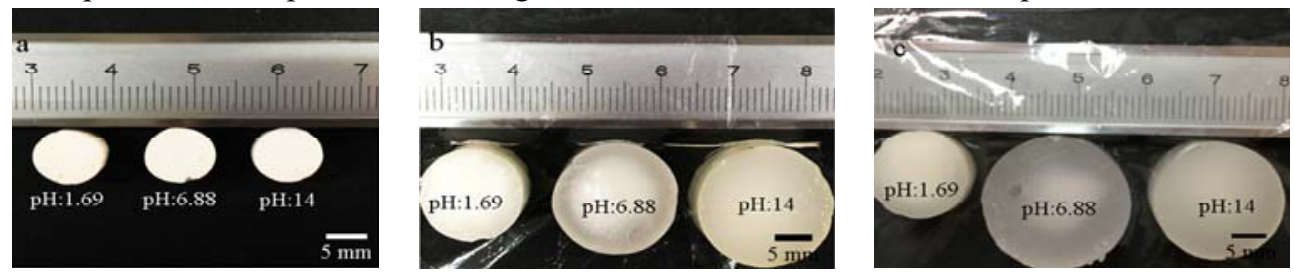

Fig.3 Digital photos of porous PAA in different $\mathrm{pH}$ respectively: (a) dry monoliths; (b) 30 min later in different $\mathrm{pH}$ aqueous; (c) $4 \mathrm{~h}$ later in different $\mathrm{pH}$ aqueous.

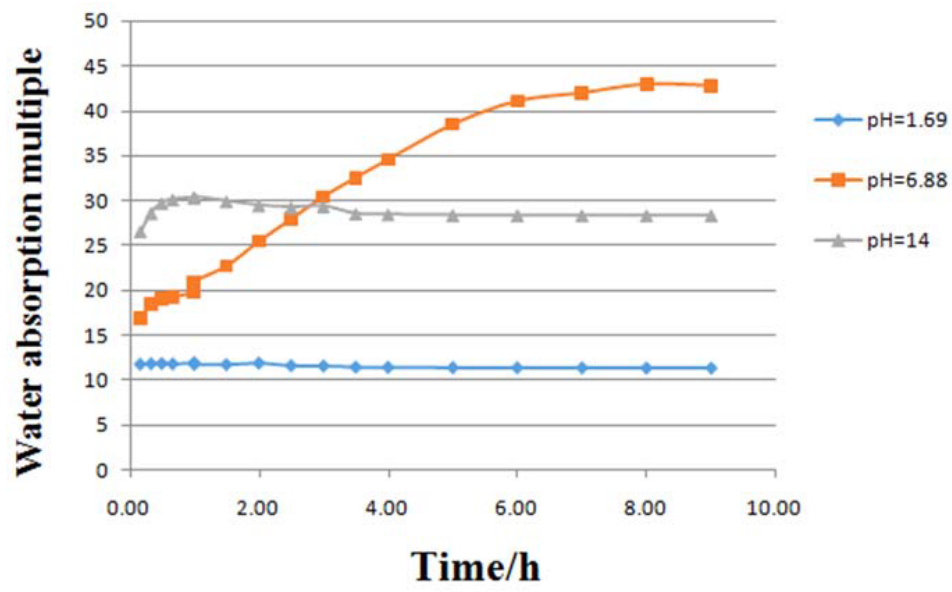

Fig.4 Curves of water absorption multiple vs time for the monoliths in different $\mathrm{pH}$ medium.

\section{Summary}

A series of porous PAA were prepared with o/w HIPEs that was stabilized with the surfactant (Tween 60) low to $0.5 \mathrm{wt} \%$. The addition of Tween 60 not only made the HIPEs stable but also determined the interconnectivity degree of resulting porous PAA. And the morphology of the porous PAA were also tailored by changing the monomer concentration and internal phase fraction. Moreover, swelling behavior of these samples were test in different $\mathrm{pH}$ aqueous solution, which demonstrated that these porous materials have a maximum swelling value in neutral water.

\section{Acknowledgements}

This research was supported by the National Natural Scientific Foundation of China 
(51203048, 51103042), the Fundamental Research Funds for the Central Universities, Innovation Program of Shanghai Municipal Education Commission (12ZZ056).

\section{References}

1. P. J. Becher, Colloid Interf. Sci., 1974, 49: 333-334.

2. P. Krajnc, D. Štefanec, I. Pulko, Macromol. Rapid Commun., 2005, 26: 1289-1293.

3. A. Menner, V. Ikem, M. Salgueiro, M.S. Shaffer, A. Bismarck, Chem. Commun., (Cambridge, England), 2007, 41: 4274-4276.

4. X. Zheng, Y. Zhang, Wang Het, Q. Du, Macromolecules, 2014,19: 6847-6855.

5. S. Zhang, J. Chen, Polymer, 2007, 48: 3021-3025.

6. A. Barbetta, N.R. Cameron, Macromolecules, 2004, 37: 3202-3213.

7. M.S. Silverstein, Prog. Polym. Sci., 2014, 39: 199-234.

8. N.R. Cameron, D.C. Sherrington, L. Albiston, D.P. Gregory, Colloid and Polym. Sci., 1996, 6: 592-595. 